\title{
ANALYTICAL REVIEW OF ADVANCE OPTICAL MODULATION FORMATS
}

\author{
Raza Ali ${ }^{1}$, Muhammad Shoaib Ali ${ }^{2}$, Talha $\mathrm{Mir}^{3}$, Bilal Shabir ${ }^{4}$ and Umar Farooq \\ Lilla $^{5}$ \\ Department of Telecommunication Engineering, BUITEMS, Quetta, Pakistan
}

\begin{abstract}
Fiber optics communication system supports the broadband data services and advance internet applications. High speed with low cost is requirement of the system. Among the other enabling technologies, Advance modulation formats has key role in designing optical communication system. This paper gives an analytical review on different modulation formats and their effectiveness.
\end{abstract}

Keyword: Optical Modulations, Optical Fiber, Optical Modulators

\section{Introduction}

In today's fast growing technical society, communication is broadening rapidly. Mobile data and voice services, multimedia broadcast system, high capacity data network and specially internet usage increasing rapidly. Internet users need to access information rapidly and amount of data flowing through the internet is very huge. These technologies are used at large extent and have their own characteristics and advantages over particular systems [1]. To compare these technologies, the most important parameter is the maximum bit rate distance (BL) IE maximum bit rate they can support without regenerating digital information.

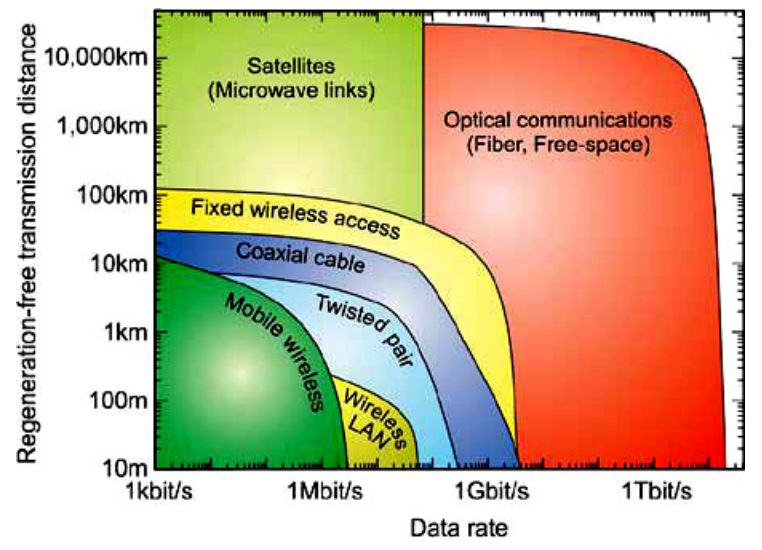

Figure 1. Comparison [1]

Figure 1. shows the comparison of different wired and wireless technologies based on this metric.It is clear from the figure that optical fiber can support higher data rates at longer distance but still not enough to support the traffic growth due to some limiting factors such as amplifier bandwidth limitation, scattering and dispersion [2]. 
Spectral efficiency can be magnified with the usage of those components that has low-loss in optics and to achieve this, optical switches and amplifiers with dispersion managing devices can be utilized. Efficiency factor can also be increased by using cutting-edge optical fibers and innovative modulation formats. In this way noise can be abated, nonlinear signal distortions reduced and high signal powers can be maintained [1].

This paper gives a comparison between advanced optical modulation formats on efficiency base at long distance. The selection of format is to reduce the linear and nonlinear attenuation in fiber before discussing the modulating formats first we will present a brief description about the nonlinear losses which are the main limiting factors.

\section{Limiting factors in optical fiber communication}

\subsection{Linear effects}

Fiber attenuation is a limiting factor for optical communication system but with improvements in manufacturing has reduced the loss up to $0.2 \mathrm{~dB} / \mathrm{km}$ at $1550 \mathrm{~nm}$.

Chromatic dispersion is a parameter which limits the spectral constituents of the signal to cover with similar speed. Components travels with different speed causes the pulses broaden effect which results bit-to-bit overlapping. Signal after detection may be degraded due to (ISI) inter symbol interference. Chromatic dispersion can be overcome through joining the transmission fiber with the dispersion fiber with reverseability [3].

Because of deficiencies in fiber manufacturing, polarization mode dispersion limits the optical communication which causes degradation in high bit rate transmissions and pulse broadening [4]. To overcome PMD number of solutions have been proposed including replacement of fiber, regeneration of optical signal before the extreme effect of PMD and to adopt the appropriate modulation format that has better safety against PMD.

For a long distance communication multiple optical amplifiers are used in series to minimize the optical signal to noise ratio. With the decrease of OSNR, BER increases which causes signal recovery difficult. To minimize this problem either FEC can be used or increasing the signal power [5].

\subsection{Nonlinear Effects}

Boosting the power of signal at the amplifier's output, introduces nonlinearity such as stimulated scattering processes, which changes the wavelength of signals and produces unwanted noise, crosstalk, and power depletion in the optical signal.

SPM (Self Phase Modulation) is a change in the phase of optical pulses due to change in refractive index of fiber. Refractive index changes in response of applied electric intensity (Kerr effect).

XPM (Cross Phase Modulation) it is a nonlinear phase shift of light when one propagating wavelength effect the phase of another co-propagating wavelength. 
Four-wave mixing (FWM) is a type of optical Kerr effect which interacts to generate new wavelength in optical signal in case of two or more wavelengths of different lights are launched in optical fiber.

\section{Modulation Technologies}

Light possesses a number of parameters that can be modulated to transmit information on an optical path. It includes intensity of light, phase, frequency and polarization of light. Intensity modulation is considered the most applicable among the others due to its simplicity of detecting the packet of photon [6]. It is mostly used in high speed optical communication. The most costeffective and basic modulation techniques are directly modulated lasers, MZMs and EAMs [1].

\subsection{Directly Intensity Modulator}

Intensity modulation is easy to implement data on an optical carrier by directly modulating the laser source. In binary intensity modulation, the information is modulated on laser that drives current, which are used to on and off the laser light. Only semiconductor lasers are of interest in direct modulation because semiconductor lasers are efficient and fulfill the bandwidth requirement required for direct modulation. The problem faced by direct modulation for long distance digital communication is frequency chirp.

\subsection{External Intensity modulator}

External modulation can overrule the problems faced by direct modulation but it is complex and expensive. The principle of operation of EAMs is absorption.

\subsection{Mach-Zehnder Intensity modulator}

Mach-Zehnder or electro-absorption modulators are mainly used for external intensity modulation. The principle of operation of MZMs is based on interference.

\section{Advance modulation formats}

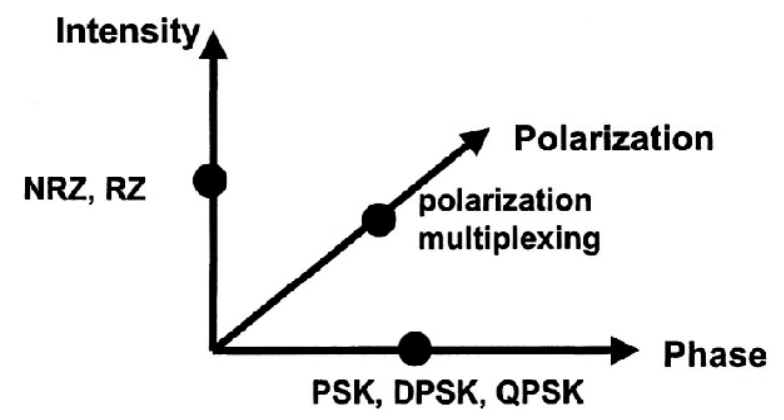

Figure 2. Modulation formats 
In optical communication transponders required to operate at a very high data rate as multi gigabits/s. A few years ago many devices were suitable for such data rate but performance is restricted due to some basic needs such as binary modulation of light. Now, today's systems are not only relying on binary OOK but also use multilevel intensity and phase modulation as well to support such high data rate. So, we use the term "advance" in conjunction with modulation formats. In optics we can modulate the light wave by changing one of the characteristics which are amplitude, phase, and polarization as shown in figure 2.Here we will focus on intensity and Phase modulation only because polarization is more attenuate is more affected by non-linear attenuation [7].

\subsection{Intensity Modulation Formats}

\subsubsection{No Return-to-Zero On/Off Keying (NRZ-OOK)}

Non-return-to-zero on-off-keying (NRZ-OOK) was one of the major modulation formats in IM fiber-optical communication systems. A number of different (NRZ) OOK codes are widely used

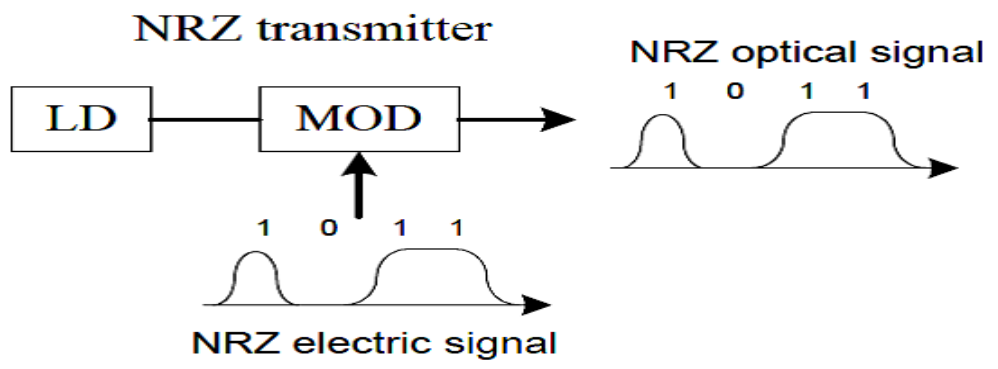

Figure 3 NRZ optical signals

over commercial systems for modulation (to achieve a high bit rate) because of their simplicity, low bandwidth requirement, immunity to laser phase noise, compatible with updated system standards and has a low implementation cost [8].Advancement in an optical communication system for high data rate at long distance, the NRZ modulation format fail to perform with its advantages on high capacity optical systems. The foremost modulation technique of IM optical fiber is still non-return-to-zero on-off-keying (NRZ-OOK).

The optical signal generation process of NRZ is shown in figure 3. Simple NRZ represents a pulse with 1 and 0 is represented as no pulse. For short distance communication DMLs and EAMs can be used to generate NRZ while for a longer distance MZMs can be useful. These modulators convert an OOK electrical signal into an OOK optical signal of same data rate. At the receiver end photodiodes are used to detect NRZ optical signal and convert into electrical current.

\subsubsection{Drawbacks}

There is no guard against inherent error monitoring, correction capabilities and no self-clocking features. For a long string of NRZ ones and zeroes shows no timing information therefore it needs a high stable clock but that will increase costs and system startup time. NRZ is more affected by ISI so for the better performance balancing must be done between noise reduction and ISI. NRZ format does not appear to be sufficiently efficient for several applications while other formats 
have been proposed to allow optical transmission over longer distances because of a better tolerance to nonlinear effects, optical noise, chromatic dispersion, PMD distortion and wider spectrum of modulated optical signal compared to NRZ.

\subsubsection{Return-to-Zero On/Off Keying (RZ-OOK)}

Return to zero-OOK formats are mostly used for long distance communication because it has a number of advantages over NRZ format. In Return-to-zero (RZ), the signal drops or returns to zero in the center of the bit period which results a stream of ones, represented as an interchanging signal. It provides clock recovery which is useful during recovery of information at the receiver

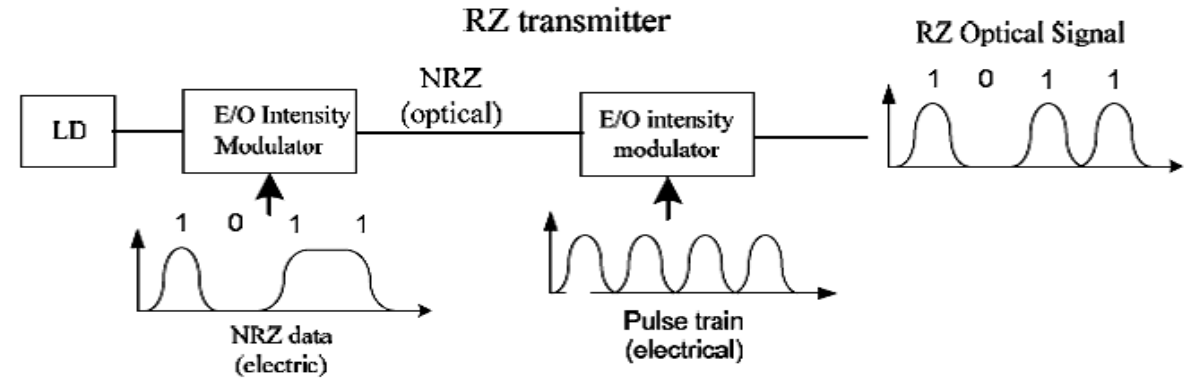

Figure 4

end. RZ provides stronger tolerance against ISI causes imperfections in frequency response and has best eye opening. RZ format also shows better tolerance against PMD, chromatic dispersion and nonlinearities because of periodic optical signal data pattern. Like NRZ, representation of the signal is same in RZ i.e. 1 is represented as a pulse for half period and 0 is represented as no pulse as shown in figure 4.To generate RZ shape of optical signal, internal intensity modulator is used to generate NRZ optical signal and modulated by a synchronized pulse train (usually a clock signal) of same data rate as the electrical signal had while generating intensity modulator [9].RZ pulses are normally generated by using an MZ modulator of sinusoidal waveform. To implement $\mathrm{RZ}$ format, RZ waveform can drive electronically as well as by artifact the output pulses from an NRZ signal through an extra modulator. Making RZ drive signal electronically modulated on optical fiber carrier (data rate up to $10 \mathrm{~Gb} / \mathrm{s}$ ) or when carving out pulses from an NRZ signal called pulse carver (data rate $40 \mathrm{~Gb} / \mathrm{s}$ or greater) [1].

\subsubsection{Drawbacks}

As compared to NRZ, RZ formats mostly require a slightly more composite transmitter structure and expensive and required high bandwidth. It has stronger signal degradation due to chromatic dispersion and has less energy per symbol than NRZ.

\subsubsection{Carrier-Suppressed Return-to-Zero (CSRZ)}

Carrier-Suppressed Return-to-Zero (CSRZ) is a multilevel modulation technique that possesses memory. The main difference between conventional RZ and CS-RZ is phase shift of $\pi$ between neighboring bits. CS-RZ has no DC components because of alternating phase in optical domain which results in zero carrier component of CS-RZ in the spectrum. The generation of CS-RZ optical signal requires two electro-optic modulators.At the start intensity modulator encodes the NRZ data which is further uses to generate its CS-RZ optical signal, secondary intensity 
modulator that modulates the optical signal. This intensity modulator is biased and provides minimum power at transmission point, driven by a sinusoidal clock, at half data rate of the electrical signal. The other MZ intensity modulator biased, doubles the frequency and change the phase of the modulating signal (output pulse train alternatively between 0 and $\pi$ ). To form this, only half data rate of bandwidth is required for the secondary electro-optical modulator to minimize its complexity. The duty cycle of MZM's generated CSRZ decreases from $67 \%$ to $50 \%$ by reducing the drive voltage swing. CSRZ signal differs from RZ standard regarding duty cycle. An improvement of CSRZ is its good transmission with high quality filtered or even VSBfiltered. CS-RZ has enhanced tolerance against chromatic dispersion, nonlinearity and can lessen the efficiency of 4 wave-mixing in WDM systems [10].

\subsubsection{Drawbacks}

As compared to other formats CSRZ has a wavelength spacing have restricted up to $100 \mathrm{GHz}$ that makes it compatible for only short-haul transmissions [11].

\subsubsection{Chirped Return-to-Zero (CRZ)}

When a waveform symbol has acceptable analog phase modulation beside real values the qualifier chirped is used for labeling the format of modulation [1]. Ultra-long-haul point to point fiber communications mostly use CRZ. The CRZ modulation format has applications in several undersea transmissions. The performance of undersea transmission systems is reduced by the nonlinear index of fiber.

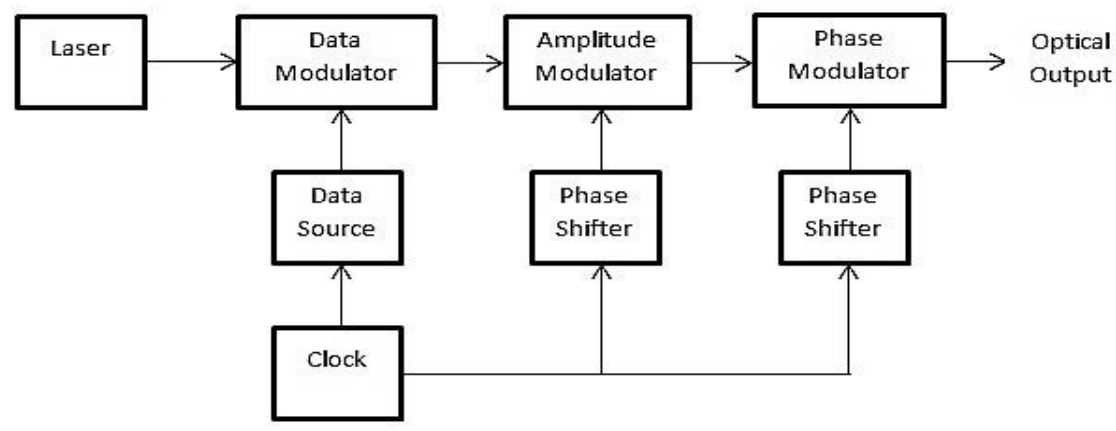

Figure 5

This is the most important factor to design an underwater optical system. CRZ signals are typically generated by transmitting NRZ waveform and sinusoidal (with the help of phase modulator) modulating the phase of the signal at the symbol rate, shown in figure 5.

This sort of three modulator transmitter architecture becomes complex and needs careful synchronization of the three driven signals. The Blend of NRZ data modulator makes RZ pulse carver and CRZ phase modulator in a single set. Dispersion compensation is one of the common techniques used for transmission system in conventional NRZ this modulation technique only operates at nearly zero average dispersion over the whole transmission medium. Dispersion compensation can be maintained by using optical gratings, dispersion-shifted fiber or optical phase conjugation. Dispersion compensation has not important in case of RZ modulation technique over a given length because this type of modulation format is less degraded than the 
NRZ modulation format by nonlinearities but the CRZ modulation format operates best with dispersion compensation and can be used to achieve propagation at greater distance [12].

Contiguous pulses significantly overlap with each other causes pulse interaction and energy exchange due to ASE noise and small nonlinearities, and makes CRZ modulation technique highly pattern dependent. By comparison to other modulation techniques such as NRZ or RZ, pulses are not strongly stretched which causes less pattern dependence as pulses do not overlap as they do in CRZ systems [6].

\subsection{Differential Phase Modulation Formats}

\subsubsection{Binary Differential Phase Shift Keying (DPSK)}

Mostly intensity modulation is used to encode data on optical signal. The phase of light can also be used to transmit the data. In DPSK modulation technique, information is transmitted by optical phase difference between two adjacent symbols. 1 is represented by a phase change of " $\pi$ " and 0 is represented by a phase change of 0 degrees [13].The intensity OOK phase modulation can be implemented by RZ and NRZ formats. DPSK formats are usually not generated by using a phase modulator although a Mach Zehnder modulator as phase is very important in this modulation. Little imperfection in phase leads the system to degrade a lot (shown in fig. 6).

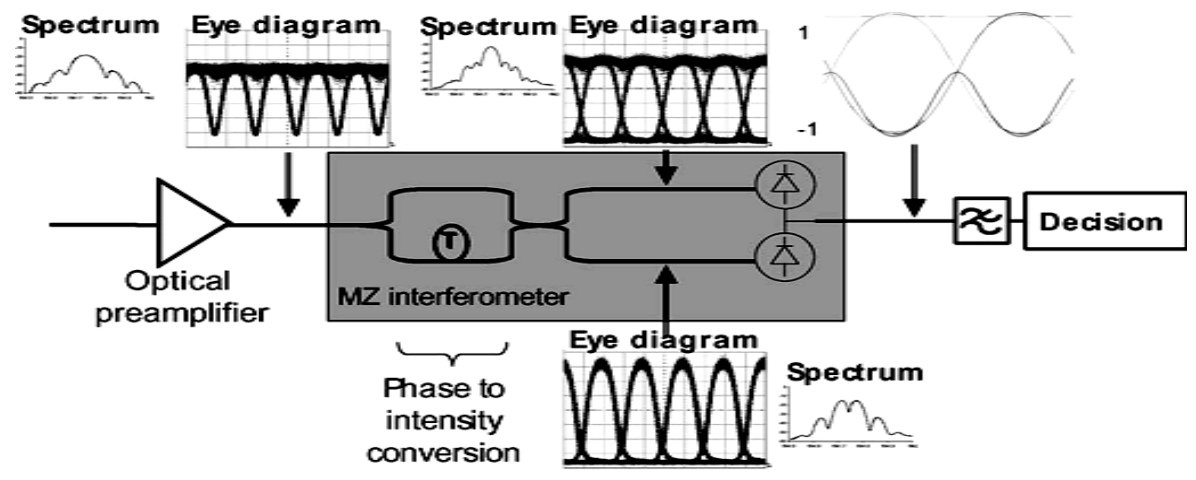

Figure : 6

While in MZM phase level is precisely defined [14]. The difference in the generation of RZ and NRZ DPASK is the use of an additional intensity modulator to generate NRZ-DPSK. At receiver Mach-Zehnder interferometer is used to detect. It splits the signal into two parts. The two parts are delayed by symbol period and recombine both parts. Here constructive and destructive outputs are formed which contains logical information but with different polarities. The main advantage of phase modulation is that it provides $3 \mathrm{~dB}$ higher sensitivity compare to OOK. We can explain this by comparing constellation diagram of both OOK and DPSK. Considering OOK 0 has zero amplitude and 1 is anywhere in circle depending upon the phase (as phase is not important here).

In DPSK 0 and 1 both have the same amplitude in order to maintain same average power but a phase difference of "pi". As the distance between 0 and 1 in case of OOK is smaller than DPSK hence the probability of detecting 1 as zero or 0 as 1 is less a DPSK, making OSNR of DPSK 


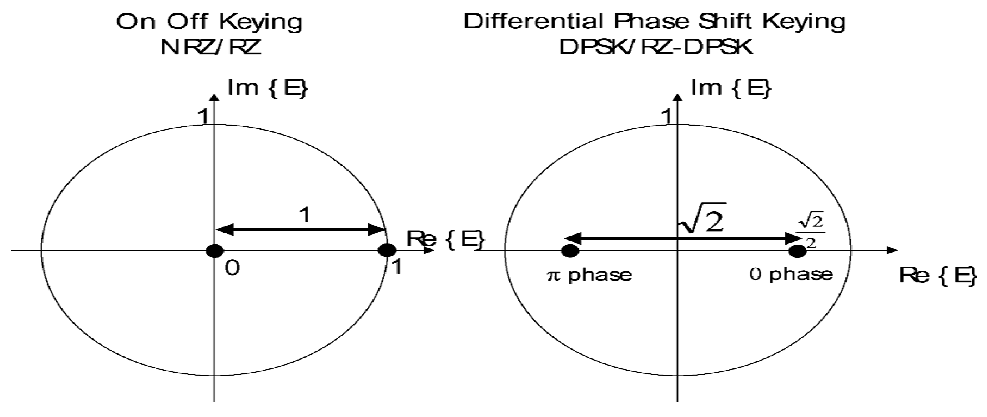

Figure : 7

better than OOK. That the symbol spacing for DPSK is increased by $\sqrt{ } 2$ compared to OOK for fixed average optical power, fig. 7. It is only concerns if DPSK is spotted by a specially designed receiver which can distinguish the phases of different symbols without the loss in power with respect to balanced configuration. But the advantages of DPSK formats are not limited to an enhanced OSNR sensitivity. It also improves tolerance of nonlinear effects. Its performance is not affected by SPM and XMP. However chromatic dispersion contributes to some level. In long distance DPSK systems, noise due to optical amplifiers is converted into phase noise using Kerr effect [11] which causes distortion in the waveform. This effect can be handled and reduced by using RZ which makes simple detection of dispersion compensation, as it as a regular bit pattern.

\subsubsection{Differential Quadrature Phase Shift Keying (DQPSK)}

At high data rate such as the 40 GB DPSK performance/s is limited due electrical receiver front end. To solve this problem DQPSK can be used as it is multi-level modulation technique (more than one bit per symbol) with low symbol rate [15]. DQPSK has attained high attention in optical networks.

DQPSK transmitter uses two phase modulator nested with phase shift, output is shifted by $\pi / 2$ when both outputs are combined. At receiver demodulation of DPQSK uses an MZI is suboptimal which results in crosstalk between two tributaries. As a result of this DQPSK has lower receiver sensitivity compare to DPSK. But it has high chromatic dispersion tolerance and high spectral Efficiency.

\section{Conclusion}

Every format has some advantages and disadvantages and selection of a particular format depends upon the design, available resources and requirement of the network. NRZ was considered as simplest format but due lack of clocks and poor performance against ISI, RZ is considered better than NRZ. RZ provides stronger tolerance against PMD, CD and nonlinearities. RZ has best eye opening. But because of the high bandwidth requirement, complex and expensive transmitter structure CS-RZ has advantage over it. More preference is given to CS-RZ optical because of its phase alternation in the optical domain and no DC component. The CRZ 
modulation technique shows excellent outcomes with dispersion compensation, therefore it is considered one of the best formats for long-haul communication undersea. Phase modulation is comparatively difficult to generate and handle but it is better than the intensity modulation. It provides better OSNR but at high data rate non-linear effects distort the signal and Optoelectronic devices do not perform well at high data rate. To solve this problem we can use DQPSK because at high data rate, DQPSK perform better than others.

\section{References}

[1] P. Winzer and René-Jean Essiambre, "Advanced Optical Modulation Formats, " Proc. IEEE, Vol. 94, No. 5, pp. 952-985May, 2006

[2] A. H. Gnauck "Advanced amplitude- and phase coded formats for $40-\mathrm{Gb} / \mathrm{s}$ fiber transmission", Proc. IEEE/LEOS Annu. Meeting, 2004

[3] Chongjin Xie, "Chromatic dispersion estimation for single-carrier coherent optical communication," IEEE Photonics Technology Letters, vol. 25, no. 10, pp. 992-995, May 2013.

[4] M. Faruk, Y. Mori, C. Zhang, and K. Kikuchi, "Proper polarization demultiplexing in coherent optical receiver using constant modulus algorithm with training mode," OptoeElectronics and Communications Conference (OECC 2010), pp. 768-769, 2010.

[5] Rasheed, I.; Abdullah, M.; Mehmood, S.; Chaudhary, M., "Analyzing the non-linear effects at various power levels and channel counts on the performance of DWDM based optical fiber communication system," Emerging Technologies (ICET), 2012 International Conference on , vol., no., pp.1,5, 8-9 Oct. 2012

[6] G. P. Agrawal Fiber-Optic Communication Systems, 2002 :Wiley

[7] Contestabile, G., Banchi, L., Presi, M., \&Ciaramella, E. Investigation of transparency of FWM in SOA to advanced modulation formats involving intensity, phase, and polarization multiplexing. Journal of Lightwave Technology, 27(19), 4256-4261. 2009

[8] D. Castagnozzi "Digital signal processing and electronic equalization (EE) of ISI", Proc. Optical Fiber Communication Conf. (OFC), 2004

[9] Abu Taher, K.; Majumder, S.P.; AzizurRahman, B.M., "Performance of multilevel modulation formats in $92 \mathrm{~Gb} / \mathrm{s}$ systems in the presence of PMD and nonlinear effects," Electrical Engineering and Information \& Communication Technology (ICEEICT), 2014 International Conference on , vol., no., pp.1,4, 10-12 April 2014

[10] Hayee, M. I., \& Haddad, R. Chirped RZ-DPSK Modulation Format in 40Gb/s WDM Transmission Systems. In Quantum Electronics and Laser Science Conference (p. JThE80).Optical Society of America. 2005

[11] H. Kogelnik "High-capacity optical communications: Personal recollections", IEEE J. Sel. Topics Quantum Electron., vol. 6, no. 6, pp.1279-1286 2000

[12] A. S. Siddiqui , S. G. Edirisinghe, J. J. Lepley, J. G. Ellison and S. D. Walker "Dispersion-tolerant transmission using a duobinary polarization-shift keying transmission scheme", IEEE Photon. Technol. Lett., vol. 14, no. 2, pp.158 -160 2002

[13] George, K. F., Bazan, T. M., Ghoniemy, S., \& El-Badawy, E. S. (2009, December). Twelve-40 Gb/s WDM communication network with different modulation formats. In High-Capacity Optical Networks and Enabling Technologies (HONET), 2009 6th International Symposium on (pp. 184188).IEEE.

[14] Kozicki, B., Takuya, O., \& Hidehiko, T. Optical performance monitoring of phase-modulated signals using asynchronous amplitude histogram analysis. Journal of Lightwave Technology, 26(10), 13531361. 2008

[15] Walsh, A.J.; Shams, H.; Mountjoy, J.; Fagan, A.; Jian Zhao; Barry, L.P.; Ellis, A.D., "Demonstrating Doubly-Differential Quadrature Phase Shift Keying in the Optical Domain," Photonics Technology Letters, IEEE , vol.25, no.11, pp.1054,1057, June1, 2013 